\title{
ON THE RELATION BETWEEN A CLUSTER SET INTRODUCED BY CONSTANTINESCU AND CORNEA, AND THE FINE CLUSTER SET OF CARTAN, BRELOT, AND NAÏM
}

\author{
H. L. Jackson \\ (received March 9, 1964)
}

The field of boundary limit theorems in analytic function theory is usually considered to have begun about 1906, with the publication of Fatou's thesis [8]. In this remarkable memoir a theorem is proved, that now bears the author's name, which implies that any bounded holomorphic function defined on the unit disk possesses an angular limit almost everywhere (Lebesgue measure) on the frontier. Outstanding classical contributions to this field can be attributed to $F$. and M. Riesz, R. Nevanlinna, Lusin, Privaloff, Frostman, Plessner, and others. During the past decade, there have been serious attempts to generalise the classical theory in such a way as to include analytic functions from a hyperbolic Riemann surface $R$ (that is, one which tolerates a Green's function) into an arbitrary Riemann surface $R^{\prime}$. The most elaborate results in this direction can be found in two papers, one by Constantinescu and Cornea [6], henceforth to be designated by $\mathrm{C}-\mathrm{C}$, and the other by Doob [7]. Since the term "cluster set" is fundamental through this discussion, we briefly define this term. Let $f$ be a function from $R$ into $R^{\prime}$ and $\mathscr{F}$ a filter of subsets of $R$. The cluster set of $f$ along $\mathscr{F}$, denoted by $\underline{C}_{\mathcal{F}}(\mathrm{f})$, is defined in such a way that $\mathrm{p}^{\prime} \in \mathrm{C}_{\mathcal{F}}(\mathrm{f})$ if and only if $p^{\prime}$ is a member of the closure of $f\left(F_{\alpha}\right)$ for every $\mathrm{F}_{\alpha} \in \mathscr{F}$.

Both C-C and Doob employ the boundary of R.S. Martin [10], as a fundamental tool, but Doob makes use of the fine cluster set of Cartan-Brelot-Naím [11], and its associated limit, whereas C-C apparently introduce their own cluster set and limit. In Math. Reviews (Vol. 23, A1025), Doob commented

Canad. Math. Bull. vol. 8, no. 1, February 1965 
that the cluster set of C-C was actually the fine cluster set of Cartan-Brelot-Naím. In this article, we shall show that the fine cluster set is always contained in the C-C cluster set, and that the two sets are identical for a continuous function. Doob's remark is therefore true for analytic functions, but not in general. For example, the C-C cluster set of a superharmonic function may strictly contain the fine cluster set. The following notation shall be employed throughout:

R, a hyperbolic Riemann surface.

$\Delta$, the Martin boundary of $R, \quad[10]$.

$1_{1}$, the subset of $\Delta$ consisting of the minimal points.

$\hat{R}$, the compact metric space consisting of the set $\mathrm{R} \cup \Delta$, endowed with the Martin metric, ([10], p. 147).

$R^{\prime}$, any Riemann surface.

$\Delta^{\prime}$, the Martin boundary of $R^{\prime}$ as defined by $C-C$ ([6], p. 44).

$\hat{R}^{\prime}$, the compact Martin space $R^{\prime} \cup \Delta^{\prime}$.

$f$ an analytic function from $R$ into $R^{\prime}$.

1. The C-C Cluster Set and Associated Limit. Let G be a region in $R, \partial G$ its frontier in $R$, and $\eta$ the identity mapping from $G$ into $R$. Following $C-C$, we define $H P(G)$ to be the set of non-negative harmonic functions on $G$.

The Set $\operatorname{HP}(\eta),([6]$, pp. 9, 10): In this section, $F$ is defined $t c$ an open subset of $G$ such that $F$ is relatively compact in $R$. We denote the frontier of $F$, (which is entirely contained in $R)$, by $\partial F$. For any $u \in H P(G)$ we define $u$ on $\partial F$ as follows:

$$
\left.\begin{array}{rl}
\underset{*}{u} & \equiv u \text { on } \partial F \cap G \\
& \equiv 0 \text { on } \partial F \cap \partial G
\end{array}\right\} .
$$


At the non-isolated points of $\partial \mathrm{F}$, the function $\mathrm{w}_{\psi^{*}}$ is lower semi-continuous and therefore resolutive ([12], p.111).

Let $\mathrm{H}_{*}^{\mathrm{F}}$ denote the generalised solution of the Dirichlet problem (Wiener function) on $\square$ which is defined by the boundary function $u$. We now define $H P(\eta)(H P(G)$ to be $\mathrm{HP}(\eta)=\left\{\mathrm{u} \in \mathrm{HP}(\mathrm{G}): \mathrm{u} \equiv \mathrm{H}_{*}^{\mathrm{F}} \mathrm{u}\right.$ on every $\left.F\right\}$.

The Operator $I_{G}([6]$, p. 18). For any $v \in H P(R)$, we define $I_{G} v=\sup \{u \in H P(\eta): u \leq v$ on $G\}$. C-C have shown that ${ }{ }_{G} V$ is itself a member of the set $\operatorname{HP}(\eta)$. The term "inverse extremalisation" employed to describe this operator comes from Kuramochi ([9], p.577).

The Set $\Delta_{1}(G) C \Delta_{1}([6]$, p. 40$)$.

Let $\hat{p} \in \Delta_{1}$, and $K_{\hat{p}}$ the associated minimal harmonic function. Following $C-C$, we define $\Delta_{1}(G)=\left\{\hat{p} \in \Delta_{1}: I_{G} K_{\hat{p}} \neq 0\right\}$. For a given $\hat{p} \in \Delta_{1}$, we define $\left\{G_{\alpha}\right\}$ to be the family of open subsets of $R$ possessing the property that $\hat{p} \in \Delta_{1}\left(G_{\alpha}\right)$ for every $G_{\alpha}$ in this family. $C-C$ have shown that $\left\{G_{\alpha}\right\}$ satisfies the axioms of a filter base on $R$ ([6], p. 25). The filter on $R$, whose base is $\left\{G_{\alpha}\right\}$, shall be denoted by the symbol $\Phi_{C-C}$.

The C-C Cluster Set $\hat{M}_{\mathrm{f}}(\hat{\mathrm{p}})([6], \mathrm{p} .46)$.

We recall that $f$ is an analytic function from a hyperbolic Riemann surface $R$ into an arbitrary Riemann surface $R^{\prime}$. Following C-C, we define $\hat{\underline{M}}_{f}(\hat{p})$ to be the cluster set (contained in $\hat{R}^{\prime}$ ) of $f$ at $\hat{p}$ along the filter $\Phi_{C-C}$. Actually $C-C$ defined $\hat{M}_{f}(\hat{p})=\bigcap_{\alpha} \overline{f\left(G_{\alpha}\right)}$ where closure is relative to $\hat{R}^{\prime}$. Henceforth, we shall refer to $\hat{M}_{f}(\hat{p})$ as the C-C cluster set of $f$ at $\hat{p}$, and in the special circumstance where $\hat{M}_{f}(\hat{p})$ reduces to a singleton subset of $\hat{R}^{\prime}$, we say that $f$ possesses a $C-C$ limit at $\hat{p}$ and denote this limit by $\hat{f}(\hat{p})$. 
2. The Fine Cluster Set of Cartan, Brelot, and Naím. While it is true that some of the following definitions were originally given in relation to classical Euclidean space, such definitions extend naturally to hyperbolic Riemann surfaces.

Thin Sets ([1], p. 327).

Let $R$ be a hyperbolic Riemann surface, $p_{0} \in R$ and $E C R$. Following Brelot, we define $E$ to be thin (effilé) at $p_{0}$ if one of the following conditions is satisfied:

(i) $\mathrm{p}_{0}$ is isolated (relative to the usual topology) with respect to the set $E \cup\left\{p_{0}\right\}$.

(ii) There exists a non-negative, superharmonic function $V(p)$ on $R$ possessing the property that $V\left(p_{0}\right)<\lim V(p)$;

(lim means limit inferior).

$$
\left\{\begin{array}{l}
p \rightarrow p_{O} \\
p \in E
\end{array}\right.
$$

Pseudo-Limit ([2], p. 29).

Brelot ([1], p.327) observed the following properties of thin sets:

(i) If $E$ is thin at $p_{0}$ and $E^{\prime} C E$ then $E^{\prime}$ is thin at $p_{0}$

(ii) If $E_{1}$ is thin at $p_{0}$, and $E_{2}$ is thin at $p_{0}$, then $E_{1} \cup E_{2}$ is also thin at $p_{0}$.

Let $\{E\}$ be the family of subsets of $R$ such that every member of $\{E\}$ is thin at $p_{O} \in R$, and $\{R-E\}$ the family of the complements of $\{E\}$. Then $\left\{R-\left(E \cup\left\{p_{0}\right\}\right)\right\}$ satisfies the axioms of a filter on $R$. Following Brelot, we define the pseudo-limit of $f$ at $p_{0}$ to be the limit of $f$ along the filter $\left\{R-\left(E \cup\left\{p_{0}\right\}\right)\right\}$ provided that such a limit exists.

The Fine Topology of H. Cartan ([5], pp. 236-237).

Cartan introduced a topology on $R$ defined to be the least topology that makes the superharmonic functions on $R$ continuous. He called this topology the fine topology, and noticed that a set 
$N C R$ is a fine neighbourhood of $p_{0} \in R$ if and only if $(R-N)$ is thin at $p_{O}$ in the sense of Brelot. The cluster set of $f$ at $p_{0}$ along the filter of deleted fine neighbourhoods is defined to be the fine cluster set of $f$ at $p_{O}$, and if the fine cluster set at $\mathrm{p}_{0}$ is a singleton subset, say $\left\{\hat{\mathrm{p}}_{0}^{\prime}\right\} C \hat{\mathrm{R}}^{\prime}$, then we say that $\hat{p}_{0}^{\prime}$ is the fine limit of $f$ at $p_{0}$. A function $f$ has a fine limit at $p_{0}$ in the sense of Cartan if and only if it has a pseudolimit at $p_{0}$ in the sense of Brelot and the two limits are equal.

The Fine Topology of L. Naim ([11], Chapt. 2).

Let $G(p, q)$ be the Green's function of $R$ and $K(p, q) \equiv \frac{G(p, q)}{G\left(p, q_{0}\right)}$ be the normalized Green's function defined on $\left(R-\left\{q_{0}\right\}\right) X R$. The Martin kernel function, denoted by $K(\hat{p}, q)$ is defined on $\left(\hat{R}-\left\{q_{0}\right\}\right) \times R$ where $K(\hat{p}, q)$ is the normalized Green's function on $\left(R-\left\{q_{0}\right\}\right) X R$. For any mass distribution $\mu$ on $R$, (a non-negative Borel measure), we define the function

$$
U(\hat{p}) \equiv \int_{R} K(\hat{p}, q) d \mu_{q}
$$

to be the Martin potential of the mass distribution $\mu$. We must keep in mind that $U$ is defined on $\hat{R} \cdot\left\{q_{0}\right\}$. On the subset $R-\left\{q_{0}\right\}$ of its domain of definition, however,

$$
U(p) \equiv \int_{R} \frac{G(p, q)}{G\left(p, q_{0}\right)} d \mu_{q} \equiv \frac{V(p)}{G\left(p, q_{o}\right)}
$$

where $\mathrm{V}(\mathrm{p})$ is the Green potential of $\mu$, and in particular a positive superharmonic function.

In her thesis, L. Nailm defined a set $E C R$ to be thin at a point $\hat{p}_{0} \in \hat{R}$ if one of the following conditions is satisfied: 
(i) $\hat{p}_{0}$ is an isolated point (in the Martin topology) of the set $E \cup\left\{\hat{p}_{0}\right\}$.

(ii) There exists a Martin potential $\mathcal{U}(\hat{\mathrm{p}})$ of some mass

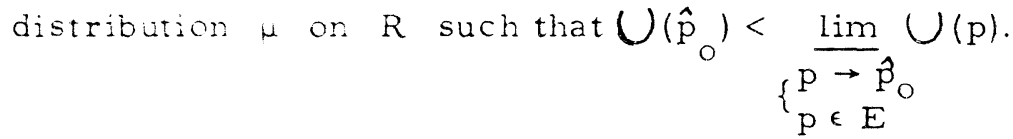

A set $E C R$ is thin at $p_{0} \in R$ in the sense of $N a i m$ if and only if it is thin there in the sense of Brelot. Nainm proved that $R$ itself is thin at $\hat{p} \epsilon \Delta$ if and only if $\hat{p}$ is not a minimal point of $\Delta$, that is $\hat{p} \in\left(\Delta-\Delta_{1}\right)([11]$, p. 25).

In order to be able to define potentials of a mass distribution or. $\hat{R}-\left\{q_{0}\right\}, \operatorname{Nallm}([11]$, pp. 28-32) introduced a new. kernel function, denoted by $\Theta(\hat{p}, \hat{q})$, defined on $\left(\hat{R}-\left\{q_{0}\right\}\right) X$ $\left(\hat{R}-\left\{q_{0}\right\}\right)$. This function has the pleasant property of being symmetric in both arguments. On the subset $\left(R-\left\{q_{0}\right\}\right) X$ $\left(R-\left\{q_{0}\right\}\right)$ of its domain of definition, $\Theta$ is defined to be

$$
\Theta(p, q) \equiv \frac{G(p, q)}{G\left(p, q_{0}\right) G\left(q, q_{0}\right)}
$$

and or $\left(\hat{R}-\left\{q_{0}\right\}\right) X\left(R-\left\{q_{0}\right\}\right), \Theta$ is defined to be

$$
\Theta(\hat{p}, q) \equiv \frac{K(\hat{p}, q)}{G\left(q, q_{0}\right)}
$$

Naim ([11], p. 30-32) shows how $\Theta$ can be extended in the second argument to $\left(\hat{R}-\left\{q_{0}\right\}\right)$ so that $\Theta(\hat{p}, \hat{q})$ is lower semicontinuous in each argument.

In the particular case where $R=\{\mathrm{z}:|\mathrm{z}|<1\}, \mathrm{q}_{0}=0$; then $G(z, 0) \equiv \log \frac{1}{|z|}, K\left(e^{i \theta}, z\right) \equiv \frac{1-|z|^{2}}{\left|e^{i \theta}-z\right|^{2}}, G(z, w) \equiv \log \left|\frac{1-\bar{w} z}{z-w}\right|$ 
Then

$$
\begin{aligned}
& \Theta(z, w) \equiv \frac{G(z, w)}{\left(\log \frac{1}{|z|}\right)\left(\log \frac{1}{|w|}\right)} \text { on }(R-\{0\}) X(R-\{0\}), \\
& \Theta\left(e^{i \theta}, w\right) \equiv \frac{K\left(e^{i \theta}, w\right)}{\log \frac{1}{|w|}} \text { on } \Delta_{1} X(R-\{0\}), \\
& \Theta\left(e^{i \theta}, e^{i \emptyset}\right) \equiv \lim _{w \rightarrow e^{i \emptyset}} \frac{K\left(e^{i \theta}, w\right)}{\log \frac{1}{|w|}} \equiv \frac{2}{\left|e^{i \theta}-e^{i \emptyset}\right|^{2}} \text { on } \Delta_{1} X \Delta_{1},
\end{aligned}
$$

For any mass distribution $\mu$ on $\hat{R}-\left\{q_{0}\right\}$, Na"im defined the function

$$
\mathrm{V}(\hat{\mathrm{p}}) \equiv \underset{\left(\hat{R}-\left\{q_{0}\right\}\right)}{ } \int \Theta(\hat{p}, \hat{\mathrm{q}}) \mathrm{d} \mu_{\hat{\mathrm{q}}}
$$

to be the $\underline{\Theta \text { potential }}$ of $\mu$ on $\hat{R}-\left\{q_{0}\right\}$. In case $\mu$ is only defined on $R-\left\{q_{0}\right\}$, then $V(\hat{p})$ is a Martin potential of some mass distribution on $R-\left\{q_{0}\right\}$.

Following Na"im ([11]), p. 40) we now define the fine topology on $\hat{R}$ to be the least topology that makes the $\Theta$ potentials continuous. The fine topology of $\mathrm{Na}^{4} \mathrm{~m}$ induces on $\mathrm{R}$ the fine topology of $H$. Cartan, and the frontier of $R$ relative to this topology is the minimal Martin boundary $\triangle_{1}$. Any set $N C R$ is a fine neighbourhood of $\hat{p} \epsilon \triangle_{1}$ if and only if $R-N$ is thin at $\hat{p}$. For an analytic function $f$ from $R$ into $R^{\prime}$ we denote the cluster set of $f$ at $\hat{p} \in \Delta_{1}$ along the filter of fine neighbourhoods as the fine cluster set of $f$ at $\hat{p}$. In case this fine cluster set reduces to a singleton subset of $\hat{R}^{\prime}$ we say that $\frac{f}{\text { possesses }}$ a fine limit at $\hat{p}$. 
3. The $\mathcal{E}$ Operator of Brelot and its Relation to the I Operator of C-C. A set E C R is defined to be polar if there exists a non-negative superharmonic function $\mathrm{V}$ on $\mathrm{R}$ such that $\lim V(q)=+\infty$ for every $p \in E$, and $V(q) \equiv+\infty$. $q \rightarrow p$

A function $f$ on $R$ that possesses a given property everywhere on $R$ except on (at most) a polar subset of $R$ is said to possess this property quasi-everywhere on $R$. For instance, we define $V$ to be quasi-superharmonic on $R$ if $V$ is superharmonic on $R$ quasi-everywhere.

Let $u \in H P(R)$, and $G$ a region in $R$. We now consider the set $\{V\}$ of positive superharmonic functions on $R$ possessing the property that every member of $\{\mathrm{V}\}$ dominates $u$ on $(R-G)$. The lower envelope of $\{V\}$ is a quasi-superharmonic function on $R$ denoted by $\Sigma_{u}^{G}([3]$, pp. 4,6).

We now define $\sum_{u}^{G} \equiv \sum_{u}^{G}$ on $S C R$ where $S$ is the set on which $\sum_{u}^{G}$ is superharmonic, $\sum_{u}^{G}(p)=\lim _{q \rightarrow p} \sum_{u}^{G}(q)$ for every $p \in R-S$.

The superharmonic function $\bar{\Sigma}_{u}^{G}$ is said to regularize $\Sigma_{u}^{G}$, and coincides with the superharmonic function $\mathcal{E}_{u}^{G}$ called the extremal of $u$ on $G$ by Brelot ([3], pp. 6, 10). The function

$$
\left.\begin{array}{rl}
\mathcal{E}_{u}^{G} & \equiv H_{*}^{\mathrm{u}}{ }^{\mathrm{G}} \text { on } \mathrm{G}, \\
& \equiv u \text { qua si-everywhere on } \mathrm{R}-\mathrm{G}
\end{array}\right\}
$$

where $\mathrm{H}_{\mathrm{u}}^{\mathrm{G}}$ is the generalized solution of the Dirichlet problem or $G$ defined by the boundary function

$$
\left\{\begin{aligned}
u=u \text { on } \partial G & \text { (boundary of } G \text { in } R \text { ) } \\
=0 \text { on } & \text { (one point compactification of } R \text { ). }
\end{aligned}\right.
$$


It is common nowadays to refer to $\mathcal{E}$ as the extremalisation operator of Brelot.

We now prove a theorem that relates the I Operator of C-C with the $\mathcal{E}$ operator of Brelot.

It is to be noted that C-C ([6], p. 21, Hilfsatz 4) have proved a similar result.

THEOREM 1. For any $u \in H P(R)$, it follows that $\mathrm{u} \equiv \mathrm{I}_{\mathrm{G}} \mathrm{u}+\mathcal{E}_{\mathrm{u}}^{\mathrm{G}}$ on $\mathrm{G}$, where $\mathrm{G}$ is any region in $\mathrm{R}$.

Proof. Let us define $\mathrm{V} \equiv \mathrm{u}-\mathcal{E}_{\mathrm{u}}^{\mathrm{G}}$ on $\mathrm{G}$, remembering that $\mathcal{E}_{\mathrm{u}}^{\mathrm{G}} \equiv \mathrm{H}_{*}^{\mathrm{u}} \mathrm{G}$ on $\mathrm{G}$, and let $F$ be an open subset of $G$ which is relatively compact in $R$. We shall first establish that $\mathrm{V} \in \mathrm{HP}(\eta)$ and hence $\mathrm{V} \leq \mathrm{I}_{\mathrm{G}} \mathrm{u}$.

We now define

$$
\left.\begin{array}{rl}
V & \equiv V \text { on } \partial F \cap G \\
& \equiv 0 \text { on } \partial F \cap \partial G
\end{array}\right\},
$$

and compare $\mathrm{V}$ with $\mathrm{H}_{\mathrm{V}}^{\mathrm{F}}$ on $\mathrm{F}$. For any regular point $p \in \partial G([4], p, 4)$ it follows that $\lim _{q \rightarrow p} H_{u}^{G}=u(p)$, and therefore $\lim V=0$. Therefore $V \equiv V$ on the regular points of $\partial F$, $q \rightarrow p$ and $V \equiv H_{V}^{F}$ on $F([4], p .1)$. It follows that $V \in H P(\eta)$, and that $\mathrm{u}-\mathcal{E}_{\mathrm{u}}^{\mathrm{G}} \leq \mathrm{I}_{\mathrm{G}} \mathrm{u}$ on $\mathrm{G}$ by definition of $\mathrm{I}_{\mathrm{G}} \mathrm{u}$.

Proceeding in the other direction, we shall establish that $E_{\mathrm{u}}^{\mathrm{G}} \leq \mathrm{u}-\mathrm{I}_{\mathrm{G}}^{\mathrm{u}}$ on $\mathrm{G}$. Since $\mathrm{I}_{\mathrm{G}} \mathrm{u} \in \mathrm{HP}(\eta)$, and since $\overline{\operatorname{Iim}} I_{G} u<+\infty$ for every $p \in \partial G$, (because $I_{G} u \leq u$ on $G$ ), $q \rightarrow p$ 
therefore $\lim { }_{G} u=0$ for every regular $p \in \partial G$ ([3], p. 10). $\mathrm{q} \rightarrow \mathrm{p}$

Hence the boundary behariour of $\left(u-I_{G} u\right)$ is the same as that of $u$ at the regular points of $\partial G$. By the (lower) extremal property of $\mathcal{E}_{u}^{\mathrm{G}}$ (and hence $\mathrm{H}_{\mathrm{u}}^{\mathrm{G}}$ ) on $\mathrm{G}$, it follow's that $\mathcal{E}_{\mathrm{u}}^{\mathrm{G}} \leq \mathrm{u}-\mathrm{I}_{\mathrm{G}}^{\mathrm{u}}$ on $\mathrm{G}$, or that $\mathrm{u}-\mathcal{E}_{\mathrm{u}}^{\mathrm{G}} \geq \mathrm{I}_{\mathrm{G}} \mathrm{u}$.

Combining this last result with an earlier one, it follows that $u \equiv I_{G} u-\mathcal{E}_{u}^{G}$ on $G$.

4. The Relation Between the C-C Cluster Set and the Fine Cluster Set. In order to prove our main result we require the following theorem of $\mathrm{Na}$ im ([11], p. 27, Theorem 5).

THEOREM 2. A set $E C R$ is thin at $\hat{p} \in \Delta_{1}$ if and only if $K_{\hat{p}} \neq \mathcal{E}_{K_{\hat{p}}}^{(R-E)}$ on $(R-E)$, where $K_{\hat{p}}$ is the minimal harmonic function associated with $\hat{p}$.

We are now in a position to prove that the $\mathrm{C}-\mathrm{C}$ cluster set always contains the fine cluster set.

THEOREM 3. The C-C cluster set contains the fine cluster set of Naím at $\hat{p} \in \wedge_{1}$ for any function $g$ from $R$ into $R^{\prime}$.

Proof. Let $G$ be any region in $R$ which is a member of the filter $\vec{\Phi}_{C}-C$ at $\hat{p}$. Then $I_{G} K_{\hat{p}} \neq 0$ on $G$, and by Theorem 1, $\mathrm{K}_{\hat{\mathrm{p}}} \neq \mathcal{E}_{\mathrm{K}_{\hat{\mathrm{p}}}}^{\mathrm{G}}$ on $\mathrm{G}$. It is a consequence of Theorem 2 that $(R-G)$ is thin at $\hat{p}$, and hence $G$ is a deleted fine neighbourhood of $\hat{p}$. It follows that the filter $\Phi_{C-C}$ at $\hat{p}$ is $\frac{\text { contained in the filter of fine neighbourhoods of } \hat{p} \text { relativized }}{\text { to } R \text {. Therefore the fine cluster set of } g \text { at } \hat{p} \text { is contained }}$ in the C-C cluster set of $g$ at $\hat{p}$.

We now observe that the two cluster sets are not in general equivalent. Let $R=\{\mathrm{z}:|\mathrm{z}|<1\}$, and $\mathrm{g}(\mathrm{z})$ be defined as follows: 


$$
\left\{\begin{aligned}
g(z) & \equiv 0 \text { on the rational points of } R, \\
& \equiv 1 \text { on the irrational points of } R
\end{aligned}\right.
$$

Since the set of rational points is thin at every boundary point $e^{i \theta}$ of $R, g$ has the fine limit 1 at $e^{i \theta}$. But the rationals are dense in $R$, so the $C$-C cluster set of $g$ at $e^{i \theta}$ is $\{0\} \bigcup\{1\}$.

We now give an example of a superharmonic function $U(z)$ on $R$, where $U(z)$ has a fine limit at $z=1$, but the C-C cluster set of $U$ at $z=1$ strictly contains the singleton fine cluster set. Let $\mu$ be a mass distribution on $R$ such that $\mu\left(\left\{q_{i}\right\}\right)=c_{i}>0$ where $q_{i}$ is an arbitrary rational point of $R$, and $\mu(\mathscr{l})=0$ where $\mathscr{l}$ is the set of irrational points of R. Then the Martin potential of this mass distribution is

$$
\bigcup(z)=\sum_{i=1}^{\infty} c_{i} K\left(z, q_{i}\right)
$$

on $R$ where $K\left(z, q_{i}\right)$ is the normalized Green's function on $R$. If $U(z) \neq+\infty$ then $U(z)$ is a superharmonic function on $R$. On the Martin boundary of $R, U\left(e^{i \theta}\right) \equiv \sum_{i=1}^{\infty} c_{i} K\left(e^{i \theta}, q_{i}\right)$ where $K\left(e^{i \theta}, q_{i}\right)$ is the Poisson Kernel function. We now choose the set $\left\{c_{i}\right\}$ such that

$$
U(1)=\sum_{i=1}^{\infty} c_{i} \frac{1-\left|q_{i}\right|^{2}}{\left|1-q_{i}\right|^{2}}
$$

is a finite number, say $A$.

Because $U(z)$ is continuous in the fine topology, therefore the fine $\lim \cup(z)=A$, or the fine cluster set of $U(z)$ at $z=1$ $z \rightarrow 1$

is $\{A\}$. Since $U\left(q_{i}\right)=+\infty$ for all rational points, therefore 
the C-C cluster set of $U(z)$ at $z=1$ is $[A,+\infty]$.

We shall now prove that if $g(p)$ is a continuous function from $R$ into $R^{\prime}$ then the fine cluster set of $g$ at $\hat{p} \epsilon \Delta_{1}$ is identical to the $C-C$ cluster set $\hat{M}_{g}(\hat{p})$.

THEOREN 4 . Let $g(p)$ be a continuous function from $R$ inc $R$. Then the fine cluster set of $g$ at $\hat{p} \epsilon \wedge_{1}$ is the $C-C$ cluster set $\hat{M}(\hat{p}) \subset \hat{R}^{\prime}$.

Proof. Let $\hat{q}^{\prime} \in \hat{M}_{g}(\hat{p})$, and $\bar{N}$ a closed neighbourhood of $\hat{c}^{\prime}$. Since $g$ is continuous, $g^{-1}(\overline{\mathbb{N}})$ is closed in $R$. If the set $\Xi^{-1}(\bar{X})$ were thin at $\hat{p}$, then $R-g^{-1}(\bar{N})$ would be a ine, oper: (usual topology) neighbourhood of $\hat{p}$, and this would contradict the definition of $\hat{q}^{\prime}$. It follows that $g^{-1}(\bar{N})$ cannot be thin at $\hat{p} \in \Delta_{1}$.

We now let $F$ be any fine neighbourhood of $\hat{p}$. Since $g^{-1}(\bar{N})$ is not thin at $\hat{p}$ it follows that $g^{-1}(\bar{N}) \cap F \neq \emptyset$. The equivalence of the two cluster sets follows immediately

\section{REFERENCES}

1. M. Brelot, Points irréguliers et transformations continues er théorie du potentiel, Jour. Math. Pures et Appl., $19(1940), 319-337$

2 - Sur les ensembles effilés, Bull. Sc. Math., $\overline{6(19.14)}, 12-36$.

3, - Minorantes sousharmoniques, extrémales et cápacités, Jour. Math. Pures et Appl., 24 (1945), 1-32.

4. A nev proof of the fundamental theorem of Kellogg-Evans or the set of irregular points in the Dirichit problem, Rend. del Circolo Mat. Palermo, Serie 2, $4(1955), 1-11$ 
5. H. Cartan, Théorie générale du balayage en potentiel Newtonien, Annales Univ. Grenoble, 22 (1946), 221-280.

6. C. Constantinescu and A. Cornea, Über das Verhalten der analytischen Abbildungen Riemannschen Flächen auf dem idealen Rand von Martin, Nagoya Math. Jour., 17 (1960), $1-87$.

7. J. L. Doob, Conformally invariant cluster value theory, Ill. Jour. Math., 5, No.4, (1961), 521-549.

8. P. Fatou, Séries trigonometriques et séries de Taylor, Acta Math., 30 (1906), 334-400.

9. Z. Kuramochi, Relations between harmonic dimensions, Proc. Jap. Acad., 30 (1944), 576-580.

10. R. S. Martin, Minimal positive harmonic functions, Trans. Amer. Math. Soc., 49 (1941), 137-172.

11. L. Naîm, Sur le rôle de la frontière de R. S. Martin dan le théorie du potentiel, Thesis, Paris, 1957.

12. M. Parreau, Sur les moyennes des fonctions harmoniques et analytiques et la classification des surfaces de Riemann, Annales Inst. Fourier Univ. Grenoble, 3 (1951), 103-197.

McMaster University

Hamilton, Ontario 